Yasunori Okabe

Nagoya Math. J.

Vol. 54 (1974), 191-213

\title{
ON THE STRUCTURE OF SPLITTING FIELDS OF STATIONARY GAUSSIAN PROCESSES WITH FINITE MULTIPLE MARKOVIAN PROPERTY
}

\author{
Dedicated to the late Machiko Okabe
}

\author{
YASUNORI OKABE
}

\section{§1. Introduction}

Let $\boldsymbol{X}=(X(t) ; t \in \boldsymbol{R})$ be a real stationary mean continuous Gaussian process with expectation zero which is purely nondeterministic. In this paper we shall investigate the structure of splitting fields of $\boldsymbol{X}$ having finite multiple Markovian property using the results in [6]. We follow the notations and terminologies in [6].

We shall remember three kinds of definitions of the $N$-ple Markovian property $(N \in N)$.

DEFINITION 1.1. We say that $\boldsymbol{X}$ has the $N$-ple Markovian property in the broad sense if the splitting field $\boldsymbol{F}_{X}^{+/-}(t)$ is generated by $N$ linearly independent random variables in $\boldsymbol{M}$ for any $t \in \boldsymbol{R}$.

It is known that $\boldsymbol{X}$ has the $N$-ple Markovian property in the broad sense if and only if $X$ has a rational spectral density of degree $2 N$ ([1], [5]).

Definition 1.2. We say that $\boldsymbol{X}$ has the $N$-ple Markovian property in the narrow sense if $\boldsymbol{X}$ has the $N$-ple Markovian property in the broad sense and $\boldsymbol{F}_{\boldsymbol{X}}^{+/-}(t)$ is equal to the germ field $\partial \boldsymbol{F}_{X}(t)$ for any $t \in \boldsymbol{R}$.

It is also known that $\boldsymbol{X}$ has the $N$-ple Markovian property in the narrow sense if and only if its spectral density is the reciprocal of a polynomial of degree $2 N$ ([1], [5], [6]).

The third definition is

Definition 1.3. We say that $\boldsymbol{X}$ has the $N$-ple Markovian property in the sense of T. Hida if, for any $N+2$ real numbers $t_{0}<t_{1}<\cdots<t_{N+1}$,

Received October 29, 1973.

This study is supported in part by Yukawa Foundation. 
$\left\{E\left(X\left(t_{n}\right) \mid \boldsymbol{F}_{\bar{X}}^{-}\left(t_{0}\right)\right) ; 1 \leq n \leq N\right\}$ is linearly independent and $\left\{E\left(X\left(t_{n}\right) \mid \boldsymbol{F}_{\bar{X}}^{-}\left(t_{0}\right)\right)\right.$; $1 \leq n \leq N+1\}$ is linearly dependent.

It is shown in [3] that, if $\boldsymbol{X}$ has the $N$-ple Markovian property in the sense of T. Hida, $\boldsymbol{X}$ has a rational spectral density of degree $2 N$.

In this paper we shall consider the case where $X$ has the $N$-ple Markovian property in the broad sense.

In $\S 2$ we shall give a formula for the canonical representation kernel of our process $X$ (Theorem 2.1). In the proof of Theorem 2.1 we shall use Theorem 8.1 in [6], which gives a formula for the canonical representation kernel of process $\boldsymbol{X}$ having the Markovian property. By the Markovian property we mean that $\boldsymbol{X}$ satisfies $\boldsymbol{F}_{\boldsymbol{X}}^{+/-}(t)=\partial \boldsymbol{F}_{\boldsymbol{X}}(t)$ for any $t \in \boldsymbol{R}$ ([5], [6]).

In $\S 3$ we shall construct an $N$-dimensional stationary Gaussian process $\mathscr{X}=(\mathscr{X}(t) ; t \in R)$ satisfying

(1.1) $\{$ the $n$-th component of $\mathscr{X}(t) ; 1 \leq n \leq N\}$ is lineary independent in $M$ and

(1.2) $\quad \boldsymbol{F}_{X}^{+/-}(t)=\boldsymbol{F}_{\mathfrak{x}}^{+/-}(t)=\sigma(\mathscr{X}(t)$ ) for any $t \in \boldsymbol{R}$ (Theorems 3.2 (ii) and 3.3). We can give an expression of the linear predictor of $X(t)(t>0)$ using the past $\boldsymbol{F}_{\bar{X}}^{-}(0)$ in terms of the process $\mathscr{X}$ (Theorem 3.2 (i)). The relation (1.2) implies that $\mathscr{X}$ has a simple Markovian property.

In $\S 4$ we shall investigate the structure of $\mathscr{X}$ from the point of view of Markov processes, and show that a Markov process $(\mathscr{X}(t)$, $\left.P(\cdot \mid \mathscr{X}(0)=x) ; t>0, x \in R^{N}\right)$ is a recurrent Gaussian diffusion process with transition probability density and has a unique invariant measure (Theorem 4.3).

We shall prove in $\S 5$ that the $N$-dimensional stationary Gaussian process $\mathscr{X}$ satisfying (1.1) and (1.2) is unique up to multiplicative nonsingular $N \times N$-matirices (Theorem 5.1).

In $\S 6$ we shall define a nonsingular $N \times N$-matrix $T$ and an associated $N$-dimensional stationary Gaussian process $\mathscr{Y}=(\mathscr{Y}(t) ; t \in R))=$ $\left(T^{-1} \mathscr{X}(t) ; t \in R\right)$. We note that the matrix $T$ can be definitely expressed in terms of the spectral density of $\boldsymbol{X}$. Then we shall prove that the $N$-th component process of $\mathscr{Y}(=Y)$ has the $N$-ple Markovian property in the narrow sense and satisfies

$$
\boldsymbol{F}_{\boldsymbol{X}}^{+/-}(t)=\boldsymbol{F}_{\boldsymbol{Y}}^{+/-}(t)=\partial \boldsymbol{F}_{\boldsymbol{Y}}(t) \quad(t \in \boldsymbol{R})
$$

(Theorem 6.2). We can also give an alternative expression of the linear 
predictor of $X(t)(t>0)$ using the past $F_{\bar{X}}^{-}(0)$ in terms of the process Y (Theorem 6.3 (i)).

Finally in $\S 7$ we shall give three applications of our results. At first we shall characterize the Markovian property of stationary Gaussian processes from the point of view of representations and then give a necessary and sufficient condition for the $N$-ple Markovian property in the sense of T. Hida (Theorems 7.1 and 7.2). Next we shall characterize the linear predictor of $X(t)(t>0)$ using the past $\boldsymbol{F}_{\vec{X}}(0)$ as a unique solution of an initial value problem of a differential equation, which is derived from the spectral density of $\boldsymbol{X}$. As the third application, we shall give an expression of nonlinear predictors of $X(t)(t>0)$ using the past $\boldsymbol{F}_{\boldsymbol{X}}^{-}(0)$ in terms of the Gaussian diffusion process $(\mathscr{X}(t), P(\cdot \mid \mathscr{X}(0)=x) ; t>0$, $x \in \boldsymbol{R}^{N}$ ) defined in $\S 4$ (Theorem 7.4).

\section{§ 2. Rational weights}

Let $N$ be a positive integer and let $\Delta=\Delta(\lambda)$ be a rational function of degree $2 \mathrm{~N}$ which is nonnegative, symmetric and integrable. Then we have the following decomposition:

$$
\left\{\begin{array}{l}
\Delta(\lambda)=\left|\frac{Q(-\lambda)}{P(-\lambda)}\right|^{2} \quad(\lambda \in \boldsymbol{R}), \\
V_{P}=C^{+}, \quad V_{Q} \subset C^{+\cup} \boldsymbol{R}, \quad V_{P} \cap V_{Q}=\phi \quad \text { and } \\
Q(z)=\sum_{n=0}^{N-1} b_{n}(-i z)^{n}, \quad P(z)=\sum_{n=0}^{N} c_{n}(-i z)^{n}, \quad b_{n}, c_{n} \in \boldsymbol{R}, c_{N} \neq 0,
\end{array}\right.
$$

where $V_{S}$ denotes the set of zero points of a polynomial $S$. Such a decomposition is unique up to multiplicative constants of absolute one.

2.1. We denote by $F$ the Fourier transform of the reciprocal of a function $P(-\cdot)$ in $(2.1)$ :

$$
F=\left(P(-\cdot)^{-1}\right)^{\wedge}
$$

It is easy to see that $F=0$ in $(-\infty, 0)$ and $F^{(n)} \in \mathscr{A}((0, \infty)) \cap L^{2}((0, \infty))$ $(n=0,1,2, \cdots)$. By Lemmas 8.5, 8.6 (ii) and Proposition 8.1 in [6] we have

LEMMA 2.1. (i ) $F^{(n)}(0+)=0(0 \leq n \leq N-1), F^{(N-1)}(0+)=2 \pi(-1)^{N} c_{N}^{-1}$, (ii) $F^{(n)} \in L^{2}(\boldsymbol{R})(0 \leq n \leq N-1)$ (distribution derivatives),

(iii) $\left\{F^{(n)} ; 0 \leq n \leq N-1\right\}$ is linearly independent in $L^{2}(\boldsymbol{R})$. 
We define for any $n \in\{0,1, \cdots, N-1\}$ an $L^{2}$-function $F_{n}$ by

$$
F_{n}(t)= \begin{cases}(2 \pi)^{-1} \sum_{k=0}^{N-n-1} c_{n+k+1}(-1)^{k+1} F^{(k)}(t) & (t>0), \\ 0 & (t \leq 0) .\end{cases}
$$

In particular we have

$$
F_{N-1}=(-2 \pi)^{-1} c_{N} F .
$$

Then it follows from Lemmas 8.2, 8.3 and Proposition 8.1 in [6] that

LeMMA 2.2. (i) $F_{0}(0+)=1, F_{n}(0+)=0(1 \leq n \leq N-1)$,

(ii) $F_{n}=(2 \pi)^{-1} \sum_{k=0}^{N-n-1} c_{n+k+1}(-1)^{k+1} F^{(k)}(1 \leq n \leq N-1)$,

(iii) $F_{0}^{(1)}=\delta-(2 \pi)^{-1} c_{0} F, F_{n}^{(1)}=-F_{n-1}-(2 \pi)^{-1} c_{n} F(1 \leq n \leq N-1)$,

(iv) $\left\{F_{n} ; 0 \leq n \leq N-1\right\}$ is linearly independent in $L^{2}(\boldsymbol{R})$.

Furthermore it follows from Theorem 8.1 in [6] that

Lemma 2.3. For any $s \in(-\infty, 0), t \in(0, \infty)$ and $n \in\{0, \cdots, N-1\}$,

(i) $F(t-s)=\sum_{n=0}^{N-1}(-1)^{n} F^{(n)}(t) F_{n}(-s)$,

(ii) $F_{n}(t-s)=(2 \pi)^{-1} \sum_{\ell=0}^{N-1}(-1)^{\ell}\left(\sum_{m=0}^{N-n-1} c_{n+m+1}(-1)^{m+1} F^{(\ell+m)}(t)\right) F_{\ell}(-s)$.

By using Lemmas 2.1 (i), 2.2 (i) and 2.2 (iii), we can show

Lemma 2.4. $\quad F_{n}^{(m)}(0+)=(-1)^{n} \delta_{m n}(0 \leq m, n \leq N-1)$.

Next we shall prove

LEMMA 2.5. There exist $N$ positive numbers $t_{0}<t_{1}<\cdots<t_{N-1}$ such that $\operatorname{det}\left(F^{(m)}\left(t_{n}\right)\right)_{0 \leq m, n \leq N-1} \neq 0$.

Proof. Assume that $\operatorname{det}\left(F^{(m)}\left(t_{n}\right)\right)=0$ for any $N$ positive numbers $t_{0}<t_{1}<\cdots<t_{N-1}$. Differentiating it $n$ times with respect to $t_{n}$ for each $n \in\{0,1, \cdots, N-1\}$ and then letting $t_{0}<t_{1}<\cdots<t_{N-1}$ tend to zero, we see from Lemma 2.1 (i) that

$$
\operatorname{det}\left(\begin{array}{lllll}
0 & & & & 1 \\
& & & \cdot & \\
& & & & *
\end{array}\right)=0
$$

This is absurd. Therefore we have the desired result.

(Q.E.D.) 
Finally in subsection 2.1 we shall show

LEMMA 2.6. The following (i) and (ii) are equivalent:

(i) $\operatorname{det}\left(F^{(m)}\left(t_{n}\right)\right)_{0 \leq m, n \leq N-1} \neq 0$ for any $N$ positive numbers $t_{0}<t_{1}<\cdots$ $<t_{N-1}$;

(ii) $V_{P} \subset\left\{z \in C^{+} ; \operatorname{Re} z=0\right\}$.

Proof. We decompose $P(z)=d_{1} \prod_{n=0}^{N-1}\left(\lambda_{n}+i z\right)$, where $d_{1}$ is a constant and $\operatorname{Re} \lambda_{n}>0(0 \leq n \leq N-1)$. Denoting by $f_{n}$ the Fourier transform of $\left(\lambda_{n}-i \cdot\right)^{-1}(0 \leq n \leq N-1)$, we find that $f_{n}(t)=2 \pi\left(\operatorname{Re}\left(\lambda_{n}\right)\right)^{-1} e^{-\lambda_{n} t}(t>0)$, $f_{n}(t)=0(t<0)$ and $F=d_{2} f_{0} * f_{1} * \cdots * f_{N-1}$ with some constant $d_{2}$. At first we assume that (ii) holds and so $\lambda_{n} \in \boldsymbol{R}(0 \leq n \leq N-1)$. We define $N+1$ functions $v_{n}$ in $\mathscr{A}((0, \infty))(0 \leq n \leq N)$ by

$$
\left\{\begin{array}{l}
v_{0}(t)=d_{1}^{-1} e^{\lambda_{0} t} \\
v_{n}(t)=e^{\left(\lambda_{n}-\lambda_{n}-1\right) t} \\
v_{N}(t)=e^{-\lambda_{N-1} t}
\end{array} \quad(1 \leq n \leq N-1),\right.
$$

and then $N$ functions $G_{n}$ in $\mathscr{A}((0, \infty))(1 \leq n \leq N)$ by

$$
G_{n}(t)=v_{N}(t) \int_{0}^{t} v_{N-1}\left(t_{1}\right) d t_{1} \int_{0}^{t_{1}} v_{N-2}\left(t_{2}\right) d t_{2} \cdots \int_{0}^{t_{N-n}} v_{n-1}\left(t_{N-n+1}\right) d t_{N-n+1} .
$$

It may be easily seen that $P\left(\frac{1}{i} \frac{d}{d t}\right) G_{n}=0$ in $(0, \infty)(1 \leq n \leq N)$. Since $v_{n}^{\prime}$ 's are positive, we can apply (II, 30) in [3] to get that $\operatorname{det}\left(G_{m}\left(t_{n}\right)\right) \neq 0$ for any $N$ positive numbers $t_{0}<t_{1}<\cdots<t_{N-1}$. Since $P\left(\frac{1}{i} \frac{d}{d t}\right) F_{n}=0$ in $(0, \infty)(0 \leq n \leq N-1)$, we see from Lemma 2.1 (iii) that there exists a nonsingular $N \times N$-matrix $C$ satisfying $\left(F^{(m)}\left(t_{n}\right)\right)=C\left(G_{m}\left(t_{n}\right)\right)$ and so (i) holds. Next let's assume that (ii) does not hold. Since $\overline{P(\lambda)}=P(-\lambda)$ $(\lambda \in R)$, we then may assume and do that $\lambda_{0} \notin R$ and $\lambda_{1}=-\overline{\lambda_{0}}$. By an easy calculation it is shown that $f \equiv f_{0} * f_{1}$ is equal to $d_{3} \sin \left(\operatorname{Re} \lambda_{0} \cdot t\right) e^{-i I_{m} \lambda_{0} \cdot t}$ in $(0, \infty)$ for some constant $d_{3}$. Since $f_{2} * f_{3} * \cdots * f_{N-1}$ is a fundamental solution of a differential operator $S\left(\frac{1}{i} \frac{d}{d t}\right)$ of order $N-2$ with constant coefficients, we find that $S\left(\frac{1}{i} \frac{d}{d t}\right) F=d_{1} f$. This implies that, for any $N$ positive numbers $t_{0}<t_{1}<\cdots<t_{N-1}$, 


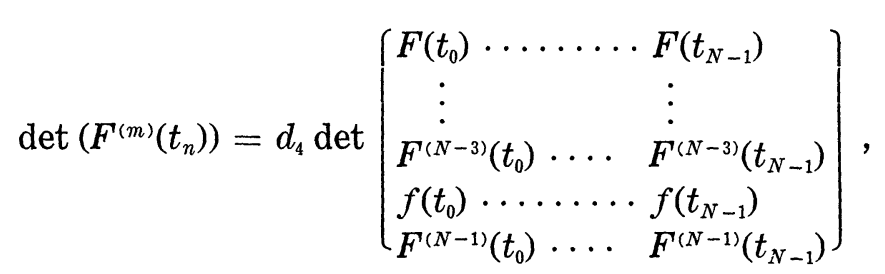

where $d_{4}$ is a constant. Since $f\left(n \pi\left(\operatorname{Re} \lambda_{0}\right)^{-1}\right)=0(n \in N)$, we find that (i) does not hold. Thus we have proved Lemma 2.6.

2.2. We denote by $E$ the Fourier transform of a function $P(-\cdot)^{-1} Q(-\cdot)$ :

$$
E=\left(P(-\cdot)^{-1} Q(-\cdot)\right)^{\wedge}
$$

By (2.2) we have

$$
E=Q\left(\frac{1}{i} \frac{d}{d t}\right) F
$$

We define for any $n \in\{0,1, \cdots, N-1\}$ an $L^{2}$-function $E_{n}$ by

$$
E_{n}(t) \equiv \begin{cases}Q\left(\frac{1}{i} \frac{d}{d t}\right) F_{n}(t) & (t>0) \\ 0 & (t \leq 0) .\end{cases}
$$

In particular we see from (2.4) and (2.6) that

$$
E_{N-1}=(-2 \pi)^{-1} c_{N} E
$$

Immediately from Lemma 2.3 and (2.7) we have

TheOREM 2.1. For any $s \in(-\infty, 0), t \in(0, \infty)$ and $n \in\{0,1, \cdots, N-1\}$,

(i) $E(t-s)=\sum_{n=0}^{N-1}(-1)^{n} F^{(n)}(t) E_{n}(-s)$,

(ii) $E_{n}(t-s)=(2 \pi)^{-1} \sum_{\ell=0}^{N-1}(-1)^{\ell}\left(\sum_{k=0}^{N-n-1}(-1)^{k+1} c_{n+k+1} F^{(k+\ell)}(t)\right) E_{\ell}(-s)$.

Moreover it follows from Lemmas 2.2 (iii) and 2.4 that

LEMMA 2.7. (i) $E_{n}(0+)=b_{n}(0 \leq n \leq N-1)$,

(ii) $E_{0}^{\prime}(t)=(-2 \pi)^{-1} c_{0} E(t), E_{n}^{\prime}(t)=-E_{n-1}(t)-(2 \pi)^{-1} c_{n} E(t) \quad(t>0,1 \leq n \leq$ $N-1)$.

Finally we shall prove

LEMMA 2.8. $\left\{E_{n}: 0 \leq n \leq N-1\right\}$ is linearly independent in $L^{2}(\boldsymbol{R})$.

Proof. Let $\alpha_{n}(0 \leq n \leq N-1)$ be real constants such that $\sum_{n=0}^{N-1} \alpha_{n} E_{n}$ 
$=0$. We then see from (2.7) that $Q\left(\frac{1}{i} \frac{d}{d t}\right)\left(\sum_{n=0}^{N-1} \alpha_{n} F_{n}\right)=0$ in $\boldsymbol{R}-\{0\}$ in the sense of distributions. Therefore, there exists a polynomial $Q_{1}$ such that $Q\left(\frac{1}{i} \frac{d}{d t}\right)\left(\sum_{n=0}^{N-1} \alpha_{n} F_{n}\right)=Q_{1}\left(\frac{1}{i} \frac{d}{d t}\right) \delta$. By taking the inverse Fourier transform of both sides, we find that $Q(-\lambda)\left(\sum_{n=0}^{N-1} \alpha_{n} \tilde{F}_{n}(\lambda)\right)=$ $Q_{1}(-\lambda)(\lambda \in R)$. Since Lemma 2.2 (ii) implies that $\tilde{F}_{n}(\lambda)=(-2 \pi)^{-1}$ $\left(\sum_{m=0}^{N-n-1} c_{n+m+1}(i \lambda)^{m}\right) P(-\lambda)^{-1}(\lambda \in \boldsymbol{R})$, there exists a polynomial $Q_{2}$ of at most degree $N-1$ such that $Q(\lambda) Q_{2}(\lambda) P(\lambda)^{-1}=Q_{1}(\lambda)(\lambda \in R)$. Hence we see from (2.1) that $Q_{2}=0$ and so $Q_{1}=0$. This implies that $\sum_{n=0}^{N-1} \alpha_{n} F_{n}=0$ and so $\alpha_{n}=0(0 \leq n \leq N-1)$ by Lemma 2.2 (iv). Thus we have proved Lemma 2.8 .

Q.E.D.

\section{§3. $\quad \boldsymbol{F}_{\boldsymbol{X}}^{+/-}(t) \quad(\mathbf{I})$}

In the sequel we shall consider a real stationary Gaussian process $\boldsymbol{X}=(X(t) ; t \in \boldsymbol{R})$ having the spectral density $\Delta$ of the form (2.1). We assume that $X$ has expectation zero. Since $P(-\cdot)^{-1} Q(-\cdot)$ is an outer function of the Hardy weight $\Delta$, we get from (2.5) the following canonical representation:

$$
X(t)=\sqrt{2 \pi}^{-1} \int_{-\infty}^{t} E(t-s) d B(s),
$$

where $(B(t) ; t \in \boldsymbol{R})$ is a standard Brownian motion satisfying

$$
\boldsymbol{F}_{\boldsymbol{X}}^{-}(t)=\sigma\left(B\left(s_{1}\right)-B\left(s_{2}\right) ; s_{1}, s_{2}<t\right) \quad \text { for any } t \in \boldsymbol{R} .
$$

Using $L^{2}$-functions $E_{n}$ in (2.7) we define random variables $X_{n}(t)$ $(t \in R, 0 \leq n \leq N-1)$ by

$$
X_{n}(t)=\sqrt{2 \pi}^{-1} \int_{-\infty}^{t} E_{n}(t-s) d B(s)
$$

and then an $N$-dimensional stationary Gaussian process $\mathscr{X}=(\mathscr{X}(t) ; t \in \boldsymbol{R})$ by

$$
\mathscr{X}(t)=\left(X_{0}(t), \cdots, X_{N-1}(t)\right)^{*} .
$$

Particularly we see from (2.8) that

$$
X_{N-1}(t)=(-2 \pi)^{-1} c_{N} X(t) \quad(t \in R) .
$$

We define an $N \times N$-matrix $A$ and an $N$-vector $\boldsymbol{b}$ by 


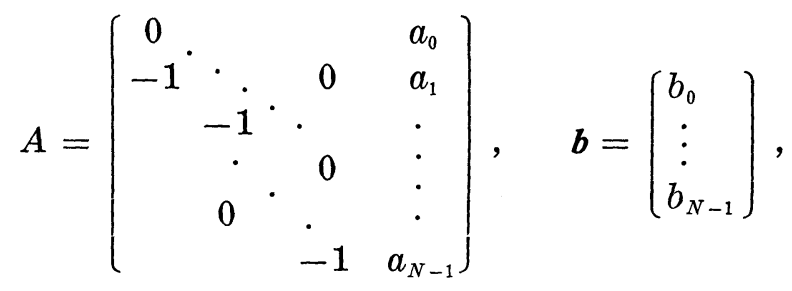

where $a_{n}=c_{n} c_{N}^{-1}(0 \leq n \leq N)$.

In the same way as Theorem 9.1 in [6] we can show from (2.8) and Lemma 2.7 that

THEOREM 3.1. For almost all $\omega$

$$
\mathscr{X}(t)-\mathscr{X}(s)=\sqrt{2 \pi}^{-1} \boldsymbol{b}(B(t)-B(s))+\int_{s}^{t} A \mathscr{X}(u) d u \quad(s<t) .
$$

In particular $\mathscr{X}(t)$ is continuous in $t \in \boldsymbol{R}$.

Noting (3.2) we see from Theorem 2.1 (i) and Lemma 2.8 that

THEOREM 3.2. (i) For any $s$ and $t \in \boldsymbol{R}, s<t$,

$$
E\left(X(t) \mid F_{X}^{-}(s)\right)=\sum_{n=0}^{N-1}(-1)^{n} F^{(n)}(t-s) X_{n}(s) .
$$

(ii) $\left\{X_{n}(t) ; 0 \leq n \leq N-1\right\}$ is linearly independent in $\boldsymbol{M}$ for any $t \in \boldsymbol{R}$.

We define for any $t \in R$ an $N \times N$-matrix $A(t)=\left(A(t)_{m n}\right)$ by

$$
A(t)_{m n}=(2 \pi)^{-1} \sum_{k=0}^{N-m-1}(-1)^{n+k+1} c_{m+k+1} F^{(n+k)}(t) \quad(0 \leq m, n \leq N-1) .
$$

Then we shall show

LEMMA 3.1. (i) For any $s$ and $t \in \boldsymbol{R}, s<t$,

$$
E\left(\mathscr{X}(t) \mid \boldsymbol{F}_{\bar{X}}^{-}(s)\right)=A(t-s) \mathscr{X}(s) .
$$

(ii) $A(t)=e^{t A}(t>0)$.

Proof. By Theorem 2.1 (ii) we have (i). We particularly see from Lemma 2.8 that $A(s+t)=A(s) A(t) \quad(s>0, t>0)$. Since $A(t)$ is continuous in $t \in(0, \infty)$ and $A(0+)=I$, this implies that there exists an $N \times N$-matrix $\tilde{A}$ satisfying $A(t)=e^{t \tilde{A}}(t>0)$. Since $B(t)-B(0)(t>0)$ are independent of $F_{\bar{X}}^{-}(0)$ and $\mathscr{X}(0)$ is $\boldsymbol{F}_{\bar{X}}^{-}(0)$-measurable by (3.2), we see from Theorem 3.1 and Lemma 3.1 (i) that 


$$
\begin{aligned}
E\left(\mathscr{X}(t) \mid F_{X}^{-}(0)\right) & =\left(I+\int_{0}^{t} A e^{u \tilde{A}} d u\right) \mathscr{X}(0) \\
& =e^{t \tilde{A} \mathscr{X}(0)} \quad(t>0)
\end{aligned}
$$

By Theorem 3.2 (ii) we get

$$
e^{t \tilde{A}}=I+\int_{0}^{t} A e^{u \tilde{A}} d u \quad(t>0) .
$$

Differentiating both sides at $t=0$, we find that $\tilde{A}=A$. Thus we have proved Lemma 3.1 .

(Q.E.D.)

In the same way as in the case of $X$, we shall consider the past fields $\boldsymbol{F}_{\mathscr{x}}^{-}(t)$, the future fields $\boldsymbol{F}_{\mathscr{x}}^{+}(t)$ and the splitting fields $\boldsymbol{F}_{\mathscr{x}}^{+/-}(t)(t \in \boldsymbol{R})$ associated with $\mathscr{X}$ (Definition 9.1 in [6]). We then see from (3.2), (3.3) and (3.4) that

$$
\boldsymbol{F}_{\bar{X}}^{-}(t)=\boldsymbol{F}_{\dot{x}}^{-}(t) \quad(t \in \boldsymbol{R})
$$

Now we shall prove the following main theorem.

THEOREM 3.3. $\boldsymbol{F}_{\boldsymbol{X}}^{+/-}(t)=\boldsymbol{F}_{\mathscr{X}}^{+/-}(t)=\sigma(\mathscr{X}(t))$ for any $t \in \boldsymbol{R}$.

Proof. By virtue of Lemma 2.5, we see from Theorem 3.2 that $\boldsymbol{M}^{+/-}(t)$ is equal to the closed linear hull of $\left\{X_{n}(t) ; 0 \leq n \leq N-1\right\}(t \in \boldsymbol{R})$. This implies by Lemma 2.1 (iii) in [6] that $F_{X}^{+/-}(t)=\sigma(\mathscr{X}(t)$ ) for any $t \in \boldsymbol{R}$. It is clear that $\sigma(\mathscr{X}(t)) \subset \boldsymbol{F}_{\mathscr{x}}^{-}(t) \cap \boldsymbol{F}_{\mathscr{x}}^{+}(t) \subset \boldsymbol{F}_{\mathscr{x}}^{+/-}(t)$ since $\mathscr{X}(t)$ is continuous in $t \in \boldsymbol{R}$. On the other hand, it follows from Lemma 3.1 that, for any $t \in R$ and any $h>0$,

$X_{n}(t+h)=A(h) \mathscr{X}(t)_{n}+\sqrt{2 \pi^{-1}} \int_{t}^{t+h} E_{n}(t+h-s) d B(s) \quad(0 \leq n \leq N-1)$.

Since $B(t+z)-B(t)(z>0)$ are independent of $\boldsymbol{F}_{x}^{-}(t)$ for any $t \in \boldsymbol{R}$ by (3.2) and (3.8), we can see that $\boldsymbol{F}_{\mathscr{x}}^{-}(t)$ is independent of $\boldsymbol{F}_{\mathscr{x}}^{+}(t)$ under the condition that $\sigma(\mathscr{X}(t))$ is known, and so that $\boldsymbol{F}_{\mathscr{x}}^{+1-}(t) \subset \sigma(\mathscr{X}(t))$. Thus we have proved Theorem 3.3.

(Q.E.D.)

\section{§4. A Gaussian diffusion process}

From Theorem 3.3 we find that a Gaussian process $(\mathscr{X}(t), P(\cdot \mid \mathscr{X}(0)$ $=x) ; t>0, x \in \boldsymbol{R}^{N}$ ) has the usual Markovian property. In this section we shall investigate several properties of such a Gaussian Markov process.

By (3.2) and Lemma 3.1 we have 
LEMmA 4.1. (i) $E_{n}(t)=\sqrt{2 \pi}^{-1}\left(e^{t A} \boldsymbol{b}\right)_{n}(t>0,0 \leq n \leq N-1)$,

(ii) $\mathscr{X}(t)=e^{(t-s) A} \mathscr{X}(s)+\sqrt{2 \pi^{-1}} \int_{s}^{t} e^{(t-u) A} b d B(u)(s<t)$.

We denote by $\mu(t, x)$ and $R(t, x)$ the mean vector and the covariance matrix, respectively, under the condition that $\mathscr{X}(0)=x\left(t>0, x \in \boldsymbol{R}^{N}\right)$ :

$$
\left\{\begin{array}{l}
\mu(t, x)=E(\mathscr{X}(t) \mid \mathscr{X}(0)=x), \\
R(t, x)=E\left(\mathscr{X}(t) \mathscr{X}(0)^{*} \mid \mathscr{X}(0)=x\right) .
\end{array}\right.
$$

It then follows from Lemma 4.1 that

$$
\left\{\begin{array}{l}
\mu(t, x)=e^{t A} x, \\
R(t, x)=R(t)=\left((2 \pi)^{-1} \int_{0}^{t} e^{s A} \boldsymbol{b}_{m} e^{s A} \boldsymbol{b}_{n} d s\right)_{0 \leq m, n \leq N-1} .
\end{array}\right.
$$

We shall prove

THEOREM 4.1. $\left\{A^{n} \boldsymbol{b} ; 0 \leq n \leq N-1\right\}$ is linearly independent.

As an application of Theorem 4.1 we find that $R(t)$ is a positive definite matrix for each $t>0$. Before the proof of Theorem 4.1, we shall prepare several lemmas.

Lemma 4.2. For any $n \in\{0,1, \cdots, N-1\}$ we set

$$
G_{n}(t)= \begin{cases}\sum_{m=0}^{N-1}(-1)^{m} b_{m} F^{(n+m)}(t) & (t>0), \\ 0 & (t \leq 0) .\end{cases}
$$

Then

$$
\left\{G_{n} ; 0 \leq n \leq N-1\right\} \quad \text { is linearly independent in } L^{2}(\boldsymbol{R}) \text {. }
$$

Proof. Let $\alpha_{n}(0 \leq n \leq N-1)$ be real constants such that $\sum_{n=0}^{N-1} \alpha_{n} G_{n}$ $=0$. We define a polynomial $S(z)=\sum_{n=0}^{N-1} \alpha_{n}(i z)^{n}$. Since $G_{m}(t)=G_{0}^{(m)}(t)$ for any $t \in R-\{0\}$, we find that $S\left(\frac{1}{i} \frac{d}{d t}\right) G_{0}=0$ in $R-\{0\}$ in the sense of distributions. Therefore, there exists a polynomial $Q_{1}$ such that $S\left(\frac{1}{i} \frac{d}{d t}\right) G_{0}=Q_{1}\left(\frac{1}{i} \frac{d}{d t}\right) \delta$ in $\boldsymbol{R}$. Noting that $G_{0} \in L^{2}(\boldsymbol{R})$ and taking the inverse Fourier transform of both sides, we find that $S(-\lambda) \tilde{G}_{0}(\lambda)=Q_{1}(-\lambda)$ $(\lambda \in \boldsymbol{R})$. On the other hand, we see that $\tilde{G}_{0}(\lambda)=Q(-\lambda) \tilde{F}(\lambda)$, since $G_{0}=$ 
$Q\left(\frac{1}{i} \frac{d}{d t}\right) F$. Hence, it follows from (2.2) that $S(\lambda) Q(\lambda)=Q_{1}(\lambda) P(\lambda)(\lambda \in \boldsymbol{R})$.

Since $S$ is a polynomial of at most degree $N-1$, this implies by (2.1) that $S=0$ and so $\alpha_{n}=0(0 \leq n \leq N-1)$. Thus we have proved Lemma 4.2 .

(Q.E.D.)

LEMMA 4.3. For any $m, n \in\{0,1, \cdots, N-1\}$ we set

$$
\gamma_{m n}=\sum_{\ell=0}^{N-1}(-1)^{\ell} b_{\ell} F_{n}^{(m+\ell)}(0+) \text {. }
$$

Then the $N \times N$-matrix $\left(\gamma_{m n}\right)_{0 \leq m, n \leq N-1}$ is nonsingular.

Proof. Differentiating (i) in Lemma $2.3 \ell+m$ times at $s=0$, we have

$$
F^{(\ell+m)}(t)=\sum_{n=0}^{N-1}(-1)^{n} F^{(n)}(t) F_{n}^{(\ell+m)}(0+) \quad(t>0,0 \leq \ell, m \leq N-1) .
$$

Multiplying it by $(-1)^{\ell} b_{\ell}$ and then summing up with respect to $\ell$, we get

$$
\sum_{\ell=0}^{N-1}(-1)^{\ell} b_{\ell} F^{(\ell+m)}(t)=\sum_{n=0}^{N-1}(-1)^{n} \gamma_{m n} F^{(n)}(t) \quad(t>0) .
$$

Therefore, by Lemmas 2.1 (iii) and 4.2, we obtain the desired result.

LEMMA 4.4. The $N \times N$-matrix $\left(E_{n}^{(m)}(0+)\right)_{0 \leq m, n \leq N-1}$ is nonsingular.

Proof. Differentiating (ii) in Theorem $2.1 \mathrm{~m}$ times at $t=0$ and then letting $s$ tend to zero, we have

$$
E_{n}^{(m)}(0+)=(2 \pi)^{-1} \sum_{\ell=0}^{N-1}(-1)^{\ell}\left(\sum_{k=0}^{N-n-1}(-1)^{k+1} c_{k+n+1} F^{(m+k+\ell)}(0+)\right) E_{\ell}(0+) .
$$

On the other hand, differentiating (i) in Lemma $2.3 m$ times and $k+\ell$ times at $t=0$ and $s=0$, respectively, we get

$$
F^{(m+k+\ell)}(0+)=\sum_{j=0}^{N-1}(-1)^{j} F^{(m+j)}(0+) F_{j}^{(k+\ell)}(0+) .
$$

Therefore it follows from Lemma 2.7 (i) that

$$
E_{n}^{(m)}(0+)=(2 \pi)^{-1} \sum_{j=0}^{N-1} \sum_{k=0}^{N-n-1} F^{(m+j)}(0+)(-1)^{j} \gamma_{k j}(-1)^{k+1} c_{k+n+1} .
$$


By Lemma 2.1 (i), the matrix $\left(F^{(m+j)}(0+)\right)_{0 \leq m, j \leq N-1}$ must be nonsingular. Therefore, we obtain the desired result noting that $c_{N}$ is not zero and using Lemma 4.3.

(Q.E.D.)

LEMMA 4.5. The $N \times N$-matrix $\left(E^{(m+n)}(0+)\right)_{0 \leq m, n \leq N-1}$ is nonsingular.

Proof. Differentiating (i) in Theorem $2.1 \ell$ times and $m$ times at $t=0$ and $s=0$, respectively, we have

$$
E^{(\ell+m)}(0+)=\sum_{n=0}^{N-1}(-1)^{n} F^{(\ell+n)}(0+) E_{n}^{(m)}(0+) .
$$

Therefore, by Lemma 4.4, we get the result.

LEMMA 4.6. $\left\{A^{n} \boldsymbol{a} ; 0 \leq n \leq N-1\right\}$ is linearly independent, where $\boldsymbol{a}=\left(a_{0} \cdots a_{N-1}\right)^{*}$.

Proof. Since $A \boldsymbol{a}=-\left(0 a_{0} \cdots a_{N_{-}}\right)^{*}+a_{N-1} \boldsymbol{a}$, we have the result noting that $a_{0}$ is not zero.

(Q.E.D.)

LEMMA 4.7. For any $\ell, m$ and $n \in\{0,1, \cdots, N-1\}$,

$$
\left(A^{n}\right)_{\ell m}=(2 \pi)^{-1} \sum_{k=0}^{N-\ell-1} c_{\ell+k+1}(-1)^{m+k+1} F^{(m+k+n)}(0+) .
$$

Proof. Differentiating $e^{t A} k$ times at $t=0$, we obtain the result from (3.7) and Lemma 3.1 (ii).

(Q.E.D.)

LEMMA 4.8. $\quad \sum_{n=0}^{N-1}(-1)^{n} b_{n} A^{n}$ is nonsingular.

Proof. We denote by $\alpha_{\ell}$ the $\ell+1$ row of the matrix $\sum_{n=0}^{N-1}(-1)^{n} b_{n} A^{n}$ and set $e_{\ell}=\left(\cdots(-1)^{n+1} c_{\ell+n+1} \cdots\right)^{*}(0 \leq \ell \leq N-1)$, where $c_{m}=0$ for $m \geq N+1$. By (2.6) and Lemma 4.7 we have

$$
\begin{aligned}
\boldsymbol{a}_{\ell} & =(2 \pi)^{-1}(-1)^{\ell} \sum_{n=0}^{N-1} \sum_{k=0}^{N-1}(-1)^{k} b_{k} F^{(\ell+k+n)}(0+) \boldsymbol{e}_{n} \\
& =\left.(2 \pi)^{-1}(-1)^{\ell} \sum_{n=0}^{N-1}\left(Q\left(\frac{1}{i} \frac{d}{d t}\right) F(t)\right)^{(\ell+n)}\right|_{t=0} \boldsymbol{e}_{n} \\
& =(2 \pi)^{-1}(-1)^{\ell} \sum_{n=0}^{N-1} E^{(\ell+n)}(0+) \boldsymbol{e}_{n} .
\end{aligned}
$$

Therefore, since $\operatorname{det}\left(\boldsymbol{e}_{0} \cdots \boldsymbol{e}_{N-1}\right)=\left((-1)^{N} c_{N}\right)^{N}$ is not zero, we have the desired result from Lemma 4.5.

After these preparations, we are in a position to prove Theorem 4.1.

Proof of Theorem 4.1.: Let $\alpha_{n}(0 \leq n \leq N-1)$ be real constants 
such that $\sum_{n=0}^{N-1} \alpha_{n} A^{n} \boldsymbol{b}=0$. Since $A \boldsymbol{b}=-\left(0 b_{0} \cdots b_{N-2}\right)^{*}+b_{N-1} \boldsymbol{a}$, we have

$$
A^{N+n} \boldsymbol{b}=(-1)^{N-1} \sum_{m=0}^{N-1}(-1)^{m} b_{m} A^{m+n} \boldsymbol{a} \quad(0 \leq n \leq N-1) .
$$

Then operating the matrix $A^{N}$ to both sides, we get

$$
\left(\sum_{m=0}^{N-1}(-1)^{m} b_{m} A^{m}\right)\left(\sum_{n=0}^{N-1} \alpha_{n} A^{n} \boldsymbol{a}\right)=\sum_{n=0}^{N-1} \alpha_{n} A^{N+n} \boldsymbol{b}=0 .
$$

and so $\alpha_{n}=0(0 \leq n \leq N-1)$ by Lemmas 4.8 and 4.6. This completes the proof of Theorem 4.1.

(Q.E.D.)

As an application of Lemma 4.4 we shall show the following

THEOREM 4.2. (i) There exist $N$ positive numbers $t_{0}<t_{1}<\cdots<t_{N-1}$ such that the matrix $\left(E^{(m)}\left(t_{n}\right)\right)_{0 \leq n \leq N-1}$ is nonsingular.

(ii) In order that for any $N$ positive numbers $t_{0}<t_{1}<\cdots<t_{N-1}$ the matrix $\left(E^{(m)}\left(t_{n}\right)\right)_{0 \leq m, n \leq N-1}$ is nonsingular, it is a necessary and sufficient condition that the zero points of $P$ are located in the positive imaginary axis.

Proof. Differentiating (i) in Theorem $2.1 \mathrm{~m}$ times at $s=0$, we have

$$
E^{(m)}(t)=\sum_{n=0}^{N-1}(-1)^{n} F^{(n)}(t) E_{n}^{(m)}(0+) \quad(t>0) .
$$

Therefore, combining Lemmas 2.5, 2.6 and 4.4, we obtain the result.

(Q.E.D.)

Now we shall apply Theorem 4.1 to get several properties of the Gaussian Markov process $\left(\mathscr{X}(t), P(\cdot \mid \mathscr{X}(0)=x) ; t>0, x \in \boldsymbol{R}^{N}\right)$. It is easy to see from (4.1) that the covariance matrices $R(t)(t>0)$ are positive definite. Therefore it follows from (4.1) that the Gaussian Markov process $\left(\mathscr{X}(t), P(\cdot \mid \mathscr{X}(0)=x) ; t>0, x \in \boldsymbol{R}^{N}\right)$ has a transition probability density $P(t, x, y)$;

$$
\left\{\begin{array}{l}
P(\mathscr{X}(t) \in d y \mid \mathscr{X}(0)=x)=P(t, x, y) d y \\
P(t, x, y)=(2 \pi)^{-N / 2}(\operatorname{det} R(t))^{-1 / 2} e^{-1 / 2\left(y-e^{t A} x, R^{-1}(t)\left(y-e^{t A} x\right)\right)}
\end{array}\right.
$$

Since $\boldsymbol{b}$ is not zero, it follows from Theorem 3.1 that

$$
\sigma(B(s)-B(t) ; s, t \in D) \subset \boldsymbol{F}_{\mathfrak{x}}(D) \quad \text { for any open set } D \text { in } \boldsymbol{R} .
$$

Therefore, by (3.2), (3.8) and (4.3), we can apply K. Ito's formula to the stochastic differential equation in Theorem 3.1 and find that the Gaussian 
Markov process $\left(\mathscr{X}(t), P(\cdot \mid \mathscr{X}(0)=x) ; t>0, x \in \boldsymbol{R}^{N}\right)$ becomes a diffusion process whose infinitesimal generator $\mathscr{G}_{\mathfrak{X}}$ is given by

$$
\mathscr{G}_{\mathfrak{X}}=\frac{1}{2}\left(\sqrt{2 \pi}{ }^{-1} \boldsymbol{b} \cdot \nabla\right)^{2}+(A x) \cdot \nabla .
$$

From Theorem 4.1 we find that this differential operator $\mathscr{G}_{\mathfrak{X}}$ is hypoelliptic ([4]).

It is easy to see from (2.1) and (3.6) that the characteristic equation of the matrix $A$ is equal to $(-1)^{N} c_{N}^{-1} P\left(i^{-1} \lambda\right)$ :

$$
\operatorname{det}(\lambda-A)=(-1)^{N} c_{N}^{-1} P\left(i^{-1} \lambda\right)=(-1)^{N} \sum_{n=0}^{N} a_{n}(-\lambda)^{n} .
$$

This particulary implies that the real part of all eigenvalues of $A$ is negative. Noting this fact and applying Theorems 4.1,6.1 and 7.1 in [2] to our Gaussian diffusion process, we have

THEOREM 4.3. The Gaussian diffusion process $(\mathscr{X}(t), P(\cdot \mid \mathscr{X}(0)=x)$; $\left.t>0, x \in \boldsymbol{R}^{N}\right)$ is recurrent and there uniquely exists an invariant measure $\mu(d y)$ :

$$
\left\{\begin{aligned}
\mu(d y) & =\varphi(y) d y, \\
\varphi(y) & =e^{-\frac{1}{2}\left(y, R^{-1}(\infty) y\right)},
\end{aligned}\right.
$$

where $R^{-1}(\infty)$ is the inverse matrix of a positive definite matrix $R(\infty)$ $=\lim _{t \rightarrow \infty} R(t)$.

Remark 4.1. It follows from (4.1) that

$$
\left.R(\infty)=\left((2 \pi)^{-1} \int_{0}^{\infty} e^{t A} \boldsymbol{b}_{m} e^{t_{A}} \boldsymbol{b}_{n} d t\right)\right)_{0 \leq m, n \leq N-1} .
$$

\section{§5. $\boldsymbol{F}_{\boldsymbol{X}}^{+1-}(t)$ (II)}

We have constructed in $\S 3$ an example $\mathscr{X}$ of $N$-dimensional stationary Gaussian processes $\mathscr{Y}=(\mathscr{Y}(t) ; t \in R)$ satisfying the following conditions :

(5.1) $\mathscr{Y}(t)$ is continuous in the mean;

(5.2) For any $t \in R$, each component of $\mathscr{Y}(t)$ belongs to $M$ and the $n$ th component of $\mathscr{Y}(t) ; 1 \leq n \leq N\}$ is linearly independent;

(5.3) $\boldsymbol{F}_{X}^{+1-}(t)=\sigma(\mathscr{Y}(t))$ for any $t \in \boldsymbol{R}$.

In this section we shall show the next theorem about the uniqueness of such a process. 
THEOREM 5.1. For any N-dimensional stationary Gaussian process $\mathscr{Y}=(\mathscr{Y}(t) ; t \in \boldsymbol{R})$ satisfying (5.1), (5.2) and (5.3), there uniquely exists a constant nonsingular $N \times N$-matrix $T$ such that $\mathscr{Y}(t)=T \mathscr{X}(t)$ for any $t \in \boldsymbol{R}$.

Before proving this theorem, we shall prepare three lemmas. We define for any $t \in \boldsymbol{R}$ an $N \times N$-matrix $K_{\mathfrak{x}}(t)$ by

$$
K_{\mathfrak{x}}(t)=E\left(\mathscr{X}(t) \mathscr{X}(0)^{*}\right) .
$$

By Theorem 3.2 (ii) and Lemma 3.1 we have

LEMMA 5.1. (i) $K_{\mathfrak{x}}(0)$ is symmetric and positive definite,

$$
K_{\mathfrak{x}}(t)= \begin{cases}e^{t A} K_{\mathfrak{x}}(0) & (t \geq 0), \\ K_{\mathfrak{x}}(0) e^{-t A} & (t<0) .\end{cases}
$$

We define a symmetric $N \times N$-matrix $B$ by

$$
B=\left(b_{m} b_{n}\right)_{0 \leq m, n \leq N-1} .
$$

Then we shall prove

LEMMA 5.2. $A K_{\mathfrak{x}}(0)+K_{\mathfrak{x}}(0) A^{*}=-(2 \pi)^{-1} B$.

Proof. Since $\mathscr{X}=(\mathscr{X}(t) ; t \in R)$ is stationary, it follows from (3.2), (3.8) and Lemma 4.1 (ii) that

$$
K_{\mathfrak{x}}(0)=e^{t A} K_{\mathfrak{x}}(0) e^{t A^{*}}+(2 \pi)^{-1} \int_{0}^{t} e^{s A} B e^{s A^{*}} d s \quad(t>0) .
$$

Differentiating it at $t=0$, we obtain the result.

(Q.E.D.)

Next we shall show the following general statement.

LEMMA 5.3. Let $A, B$ and $K$ be real $N \times N$-matrices such that

(i) $B=\left(b_{m} b_{n}\right)_{0 \leq m, n \leq N-1}, \quad \boldsymbol{b}=\left(b_{0} \cdots b_{N-1}\right)^{*} \neq 0$,

(ii) $K$ is symmetric and positive definite,

(iii) $A K+K A^{*}=-B$

and

(iv) $\left\{A^{n} \boldsymbol{b} ; 0 \leq n \leq N-1\right\}$ is linearly independent.

If an $N \times N$-matrix $\tilde{A}$ satisfies

$$
e^{t \tilde{A}} K e^{t \tilde{A}^{*}}=e^{t A} K e^{t A^{*}} \quad \text { for any } t \in \boldsymbol{R},
$$

then

$$
\tilde{A}=A
$$


Proof. Since $K$ has a symmetric and positive definite root $K^{\frac{1}{2}}$, we can define $A_{1}=K^{-\frac{1}{2}} A K^{\frac{1}{2}}, \tilde{A}_{1}=K^{-\frac{1}{2}} \tilde{A} K^{\frac{1}{2}}$ and $B_{1}=K^{-\frac{1}{2}} B K^{-\frac{1}{2}}$. It then follows that

$$
\left\{\begin{array}{l}
A_{1}+A_{1}^{*}=-B_{1}, \\
e^{t \tilde{A}_{1}} e^{t{\tilde{A_{1}}}_{1}^{*}}=e^{t A_{1}} e^{t A_{1}^{*}}
\end{array} \quad \text { for any } t \in \boldsymbol{R} .\right.
$$

Since $B_{1}$ is a symmetric, nonnegative definite matrix of rank one, there exist an orthogonal matrix $P_{1}$ and a positive number $\varepsilon$ such that $B_{1}=$ $P_{1}\left(\begin{array}{lll}\varepsilon_{0} & & 0 \\ & \ddots & \\ 0 & & 0\end{array}\right) P_{1}^{-1}$ and so $\varepsilon^{-1} \sum_{n=0}^{N-1}\left(B_{1}\right)_{n n}=1$. Therefore we can find another orthogonal matrix $P_{2}$ such that $\left(P_{2}\right)_{n 0}=\sqrt{\varepsilon^{-1}}\left(K^{-\frac{1}{b}} b\right)_{n}(0 \leq n \leq N-1)$, because $\left(B_{1}\right)_{n n}=\left(K^{-\frac{1}{b}} \boldsymbol{b}\right)_{n}^{2}$. It is then easy to see that $P_{2}\left(\begin{array}{lll}\varepsilon_{0} & & 0 \\ & \ddots & \\ 0 & & 0\end{array}\right) P_{2}^{-1}=$ $B_{1}$. Hence, setting $A_{2}=P_{2}^{-1} A_{1} P_{2}, \tilde{A}_{2}=P_{2}^{-1} \tilde{A}_{1} P_{2}$ and $T=\left(\begin{array}{llll}-\varepsilon_{0} & & 0 \\ & \ddots & \\ 0 & & 0\end{array}\right)$, we see from (5.6) and Theorem 4.1 that

$$
\begin{gathered}
A_{2}+A_{2}^{*}=T, \\
e^{t \tilde{A}_{2}} e^{t \tilde{A}_{2}^{*}}=e^{t A_{2}} e^{t A_{2}^{*}} \quad \text { for any } t \in \boldsymbol{R}
\end{gathered}
$$

and

$$
\left\{\left(\left(A_{2}^{n} T\right)_{00},\left(A_{2}^{n} T\right)_{10}, \cdots,\left(A_{2}^{n} T\right)_{N-10}\right)^{*} ; 0 \leq n \leq N-1\right\}
$$

is linearly independent.

We define a sequence $\left(D_{p}\right)_{p=0}^{\infty}$ of $N \times N$-matrices by

$$
D_{p}=A_{2} D_{p-1}+D_{p-1} A_{2}^{*} \quad(p=1,2, \cdots), D_{0}=I .
$$

Since $D_{1}=T$ by (5.7), we have

$$
D_{p+1}=\sum_{k=0}^{p}\left(\begin{array}{l}
p \\
k
\end{array}\right) A_{2}^{k} T A_{2}^{* p-k} \quad(p=0,1,2, \cdots) .
$$

Setting $L=\tilde{A}_{2}-A_{2}$ and then differentiating (5.8) at $t=0$, we get

$$
L D_{p}+D_{p} L^{*}=0 \quad(p=0,1,2, \cdots) .
$$


Therefore, putting $S=\left[L, A_{2}\right]\left(=L A_{2}-A_{2} L\right)$, we see from (5.10) and (5.12) that

$$
S D_{p}+D_{p} S^{*}=0 \quad(p=0,1,2, \cdots) .
$$

From (5.12) in the case of $p=1$ we have

$$
L+L^{*}=0 \text {. }
$$

Furthermore, applying (5.12) in the case of $p=1$, we find that $[L, T]$ $=0$. Therefore, since $T=\left(\begin{array}{ccc}-\varepsilon_{0} & & 0 \\ & \ddots & \\ 0 & & 0\end{array}\right)$, we get

$$
L T=T L=0 .
$$

Similarly it follows from (5.13) in the case of $p=0$ and $p=1$ that

$$
S+S^{*}=0
$$

and

$$
S T=T S=0 .
$$

Fixing any $p_{0} \in\{0,1,2, \cdots\}$ we shall assume that $S A_{2}^{p} T=T A_{2}^{p} S=0$ for any $p \in\left\{0, \cdots, p_{0}\right\}$. By (5.7), (5.11), (5.13), (5.16) and (5.17), we find that $S A_{2}^{p_{0}+1} T=T A_{2}^{p_{0}+1} S . \quad$ Since $T=\left(\begin{array}{ccc}-\varepsilon_{0} & & 0 \\ & \ddots & \\ 0 & & 0\end{array}\right)$, this implies that $\left(S A_{2}^{p_{0}+1}\right)_{n 0}$ $=0$ for any $n \in\{1,2, \cdots, N-1\}$. Moreover we see that $\left(S A_{2}^{p_{0}+1}\right)_{00}=0$ because $S_{0 n}$ for any $n \in\{0,1, \cdots, N-1\}$ by (5.17). For this reason it follows that $S A_{2}^{p_{0}+1} T=T A_{2}^{p_{0}+1} S=0$. By mathematical induction on $p_{0}$, we conclude that $S A_{2}^{p} T=0$ for any $p \in\{0,1,2, \cdots\}$. Therefore, using (5.9), we find that $S=0$. Since this conclusion implies that $L$ commutes with $A_{2}$, it follows from (5.15) that $L A_{2}^{p} T=0$ for any $p \in\{0,1, \cdots\}$. Consequently, using (5.9) again, we see that $L=0$ and so $\tilde{A}=A$. Now we complete the proof of Lemma 5.3.

After these preparations, we are in a position to prove Theorem 5.1.

Proof of Theorem 5.1: Since the subspace of $M$ whose elements are $\boldsymbol{F}_{X}^{+/-}(t)$-measurable is equal to the space $\boldsymbol{M}^{+/-}(t)$ with the algebraic dimension $N$, it follows from (5.2) and (5.3) that there exists a nonsingular $N \times N$-matrix $T(t)$ satisfying $\mathscr{Y}(t)=T(t) \mathscr{X}(t)(t \in R)$. For any 
$s$ and $t \in R, s<t$, we define an $N \times N$-matrix $C(t, s)$ by

$$
C(t, s)=T(t) e^{(t-s) A} T(s)^{-1} .
$$

Then it follows from Lemma 3.1 and (5.2) that

$$
C(u, s)=C(u, t) C(t, s) \quad(s<t<u)
$$

and

$$
E\left(\mathscr{Y}(t) \mid \boldsymbol{F}_{\bar{X}}(s)\right)=C(t, s) \mathscr{Y}(s) \quad(s<t) .
$$

Since $\mathscr{Y}=(\mathscr{Y}(t) ; t \in R)$ is stationary, we see from (5.2) and (5.19) that $C(t, s)=C(t-s, 0)(s<t)$. Setting $C(t)=C(t, 0)(t>0)$, we can show from (5.1), (5.2) and (5.18) that $C(t)$ is continuous in $t \in[0, \infty), C(0)=I$ and $C(s+t)=C(s) C(t)(s, t \in[0, \infty))$. Therefore, there exists an $N \times N$ matrix $\tilde{A}$ such that $C(t)=e^{t T(0) \tilde{A} T(0)-1}(t \geq 0)$. Since it is easily seen that $T(t)$ is real analytic in $t \in R$, we obtain

$$
T(t)=T(0) e^{t \tilde{A}} e^{-t A} \quad \text { for any } t \in \boldsymbol{R} .
$$

On the other hand, by Lemma 5.1 and (5.19), we have

$$
C(t-s) T(0) K_{\mathfrak{x}}(0) T(0)^{*}=T(t) e^{(t-s) A} K_{\mathfrak{x}}(0) T(s)^{*} \quad(s<t) .
$$

Combining this with (5.20), we get

$$
e^{t \tilde{A}} K_{\mathfrak{X}}(0) e^{t \tilde{A}^{*}}=e^{t A} K_{\mathfrak{X}}(0) e^{t A^{*}} \quad(t \in \boldsymbol{R}) .
$$

Therefore, by Theorem 4.1, Lemmas 5.1 (i) and 5.2, we can apply Lemma 5.3. to obtain the conclusion.

(Q.E.D.)

EXAMPLE 6.1. Using $N$ positive numbers $t_{n}$ in Lemma 2.5, we define a nonsingular $N \times N$-matrix $T=\left((-1)^{n} F^{(m)}\left(t_{n}\right)\right)_{0 \leq m, n \leq N-1}$ and a stationary Gaussian process $\mathscr{Y}=(\mathscr{Y}(t) ; t \in R)=(T \mathscr{X}(t) ; t \in \boldsymbol{R})$. It follows from Theorem 3.2 (i) that the $n+1$-th component of $\mathscr{Y}(t)$ is equal to $E\left(X\left(t+t_{n}\right) \mid \boldsymbol{F}_{\bar{X}}^{-}(t)\right)(t \in R, 0 \leq n \leq N-1)$.

\section{§6. $F_{X}^{+/-}(t)$ (III)}

Using the $L^{2}$-function $F$ in (2.2) and the Brownian motion $B$ in (3.1), we define a real stationary Gaussian process $Y=(Y(t) ; t \in R)$ such that

$$
Y(t)=\sqrt{2 \pi^{-1}} \int_{-\infty}^{t} F(t-s) d B(s) \quad(t \in R) .
$$


It is easy to see that this representation is canonical and $\boldsymbol{Y}$ has the $\mathrm{N}$ ple Markovian property in the narrow sense. Since $Q$ is a polynomial of at most degree $N-1$, we see from Lemma 2.1 (i), (2.6) and (3.1) that

$$
X(t)=Q\left(\frac{1}{i} \frac{d}{d t}\right) Y(t) \quad(t \in \boldsymbol{R}) .
$$

Now we define an $N \times N$-matrix $T$ by

$$
T=\left(\boldsymbol{b}(-A) \boldsymbol{b} \cdots(-A)^{N-1} \boldsymbol{b}\right),
$$

which is nonsingular by virtue of Theorem 4.1. Since the characteristic polynomial of $A$ is $(-1)^{N} c_{N}^{-1} P\left(i^{-1} \lambda\right)$, it follows from Caley-Hamilton's theorem that $\sum_{n=0}^{N} a_{n}(-A)^{n}=0 \quad((4.5))$. Therefore we can easily see that

$$
T^{-1} \boldsymbol{b}=(10 \cdots 0)^{*}
$$

and

$$
T^{-1} A T=A .
$$

Using this matrix $T$ we define an $N$-dimensional stationary Gaussian process $\mathscr{Y}=(\mathscr{Y}(t) ; t \in \boldsymbol{R})$ satisfying (5.1), (5.2) and (5.3) as follows:

$$
\mathscr{Y}(t)=T^{-1} \mathscr{X}(t) \quad(t \in R) .
$$

We denote by $Y_{n}(t)$ the $n+1$-th component of $\mathscr{Y}(t)(0 \leq n \leq N-1, t \in \boldsymbol{R})$. By (2.3), (3.3), (3.7), Lemma 3.1 (ii) and 4.1 (i), we can show that

$$
\mathscr{Y}(t)=\sqrt{2 \pi}^{-1} \int_{-\infty}^{t} e^{(t-s) A}\left(\begin{array}{c}
1 \\
0 \\
\vdots \\
0
\end{array}\right) d B(s) \quad(t \in \boldsymbol{R})
$$

and

$$
\left[e^{t A}\left(\begin{array}{c}
1 \\
0 \\
\vdots \\
0
\end{array}\right)\right]_{n}=F_{n}(t) \quad(t>0,0 \leq n \leq N-1) .
$$

By (2.4), we particularly find 


$$
Y_{N-1}(t)=(-2 \pi)^{-1} c_{N} Y(t) \quad(t \in R) .
$$

By (3.8) and (6.6) we note

$$
\boldsymbol{F}_{\bar{X}}^{-}(t)=\boldsymbol{F}_{\mathscr{Q}}^{-}(t) \text {. }
$$

Using Theorem 3.1, Lemmas 3.1 and 4.1 (ii), we see from (6.4) and (6.5) that

THEOREM 6.1. For almost all $\omega$

( i ) $\mathscr{Y}(t)-\mathscr{Y}(s)=\sqrt{2 \pi}^{-1}(B(t)-B(s), 0, \cdots, 0)^{*}+\int_{s}^{t} A \mathscr{Y}(u) d u(s<t)$,

(ii) $\mathscr{Y}(t)=e^{(t-s) A} \mathscr{Y}(s)+\sqrt{2 \pi}^{-1} \int_{s}^{t} e^{(t-s) A}\left(\begin{array}{c}1 \\ 0 \\ \vdots \\ 0\end{array}\right) d B(u)(s<t)$,

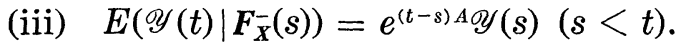

Noting (3.6) we can show from (6.6), (6.9) and Theorem 6.1 (i) that

$$
\boldsymbol{F}_{g x}(D)=\boldsymbol{F}_{\mathscr{y}}(D)=\boldsymbol{F}_{\boldsymbol{Y}}(D) \quad \text { for any open set } D \text { in } \boldsymbol{R}
$$

and

$$
\boldsymbol{F}_{\boldsymbol{Y}}^{+/-}(t)=\partial \boldsymbol{F}_{\boldsymbol{Y}}(t) \quad \text { for any } t \in \boldsymbol{R} .
$$

Therefore, combining these with Theorem 3.3, we get

THEOREM 6.2.

$$
\boldsymbol{F}_{X}^{+/-}(t)=\boldsymbol{F}_{\mathscr{y}}^{+1-}(t)=\sigma(\mathscr{Y}(t))=\boldsymbol{F}_{\boldsymbol{Y}}^{+/-}(t)=\partial \boldsymbol{F}_{\boldsymbol{Y}}(t) \quad \text { for any } t \in \boldsymbol{R} .
$$

Finally we shall give an alternative expression of the linear predictor by using the process $\mathscr{Y}$.

THEOREM 6.3. (i) For any $s$ and $t \in R, s<t$,

$$
E\left(X(t) \mid F_{\bar{X}}^{-}(s)=\sum_{n=0}^{N-1}(-1)^{n} E^{(n)}(t-s) Y_{n}(s) .\right.
$$

(ii) $\left\{Y_{n}(t) ; 0 \leq n \leq N-1\right\}$ is linearly independent in $M$ for any $t \in \boldsymbol{R}$.

Proof. By Theorem 3.2 (i) and (6.6) we have (ii). It follows from Theorem 2.1 (i) and Lemma 4.1 (i) that

$$
E(t-s)=\sum_{\ell=0}^{N-1}(-1)^{\ell} F^{(\ell)}(t)\left(e^{-s A} \boldsymbol{b}\right)_{\ell} \quad(s<0, t>0) .
$$


Differentiating both sides $n$ times at $s=0$, we get

$$
E(t)=\sum_{\ell=0}^{N-1}(-1)^{\ell} F^{(\ell)}(t)\left(A^{n} \boldsymbol{b}\right)_{\ell} \quad(0 \leq n \leq N-1) .
$$

Therefore, by Theorem 3.2 (i) and (6.6), we obtain (i).

(Q.E.D.)

\section{§ 7. Applications}

\subsection{Markovian property.}

At first we shall characterize the Markovian property of stationary Gaussian processes from the point of view of representations. In [6] we have proved

THEOREM 7.1. ([6]) In order that a real mean continuous, purely nondeterministic stationary Gaussian process $\boldsymbol{X}$ has the Markovian property:

$$
\boldsymbol{F}_{\boldsymbol{X}}^{+/-}(t)=\partial \boldsymbol{F}_{X}(t) \quad \text { for any } t \in \boldsymbol{R},
$$

it is a necessary and sufficient condition that there exists a canonical representation $\left(\sqrt{2 \pi}^{-1} E(t), B(t)\right)$ possesing

$$
\sigma(B(s)-B(t) ; s, t \in D) \subset \boldsymbol{F}_{X}(D) \quad \text { for any open set } D \text { in } \boldsymbol{R} .
$$

We shall give another proof of Theorem 7.1 in case $\boldsymbol{X}$ has a rational spectral density $\Delta$ of the form (2.1). Now let's assume (7.2). It then follows from (3.5), (3.6) and Theorem 3.1 that $\mathscr{X}(t)$ is $\partial \boldsymbol{F}_{X}(t)$-measurable for any $t \in \boldsymbol{R}$. Therefore, by Theorem 3.2 (i), we find that $E\left(X(u) \mid \boldsymbol{F}_{\bar{X}}^{-}(t)\right)$ is $\partial \boldsymbol{F}_{\boldsymbol{X}}(t)$-measurable $(t<u)$ and so that (7.1) holds. Conversely let's assume (7.1). It then follows from Lemma 2.5 and Theorem 3.2 (i) that $\mathscr{X}(t)$ is $\partial \boldsymbol{F}_{\boldsymbol{X}}(t)$-measurable for any $t \in \boldsymbol{R}$. Therefore, by (3.6) and Theorem 3.1, we obtain (7.2) since $\boldsymbol{b}$ is not zero.

Next we shall characterize the $N$-ple Markovian property in the sense of T. Hida ([3]). Immediately from Lemma 2.6 and Theorem 3.2 (i) we can show

THEOREM 7.2. In order that a real mean continuous, purely nondeterministic stationary Gaussian process $\boldsymbol{X}$ has the $\mathrm{N}$-ple Markovian property in the sense of T. Hida, it is a necessary and sufficient condition that $\boldsymbol{X}$ has a rational spectral density $\Delta$ of the form (2.1) with an additional property 


$$
V_{p}^{*} \subset\left\{z \in C^{+} ; \operatorname{Re} z=0\right\}
$$

\subsection{Initial value problem.}

We shall characterize the linear predictor using the past as a unique solution of an initial value problem. We define an $N \times N$-matrix $D=$ $\left(D_{m n}\right)_{0 \leq m, n \leq N-1}$ by

$$
D_{m n}=(-1)^{n} E^{(m+n)}(0+),
$$

which is nonsingular by Lemma 4.5.

THEOREM 7.3. We denote by $Z(t, \omega)$ the linear predictor of $X(t)$ using the whole past;

$$
Z(t, \omega)=E\left(X(t) \mid F_{X}^{-}(0)\right) \quad(t>0) .
$$

Then, for almost all $\omega \in \Omega, Z(t, \omega)(t>0)$ is a unique solution of the following initial value problem (7.5):

$$
\left\{\begin{array}{l}
Z(\cdot, \omega) \in \mathscr{A}((0, \infty)) \cap L^{2}((0, \infty)) \\
P\left(\frac{1}{i} \frac{d}{d t}\right) Z(t, \omega)=0 \quad \text { in }(0, \infty), \\
Z^{(n)}(0+, \omega)=(D \mathscr{Y}(0))_{n} \quad(0 \leq n \leq N-1) .
\end{array}\right.
$$

Proof. Since $F^{(n)} \in \mathscr{A}((0, \infty)) \cap L^{2}((0, \infty)) \quad(n=0,1,2, \ldots)$ and $P\left(\frac{1}{i} \frac{d}{d t}\right) F=0$ in $(0, \infty)$, it follows from Theorem 2.1 (i) that $E^{(n)} \in \mathscr{A}$ $((0, \infty)) \cap L^{2}((0, \infty))$ and $P\left(\frac{1}{i} \frac{d}{d t}\right) E^{(n)}=0$ in $(0, \infty)(n=0,1,2, \cdots)$. Therefore, by Theorem 6.3 (i), we have (7.5). It is clear that $Z(\cdot, \omega)$ is a unique solution of (7.5), because $P$ is a polynomial of degree $N$.

Remark 7.1. By Theorem 6.3 (ii) we note that $\left\{(D \mathscr{Y}(0))_{n} ; 0 \leq n \leq\right.$ $N-1\}$ is linearly independent.

\subsection{Nonlinear prediction.}

As the last application, we shall give an expression of nonlinear predictors of $X(t)$ using the past $F_{X}^{-}(0)$ in terms of the transition probability density $P(t, x, y)$ of the Gaussian diffusion process $(\mathscr{X}(t), P(\cdot \mid \mathscr{X}(0)$ $\left.=x) ; t>0, x \in R^{N}\right)$. Immediately from (3.5), Theorem 3.3 and $(4,2)$ we have 
THEOREM 7.4. For any bounded measurable function $f$ (or any polynomial) on $\boldsymbol{R}$ and any $t>0$,

$$
E\left(f(X(t)) \mid \boldsymbol{F}_{X}^{-}(0)\right)=\int_{R^{N}} f\left(-2 \pi c_{N}^{-1} y_{N-1}\right) P(t, \mathscr{X}(0), y) d y_{0} \cdots d y_{N-1} .
$$

\section{REFERENCES}

[1] J. L. Doob: The elementary Gaussian processes, Ann. Math. Statist. 15 (1944), $229-282$.

[2] H. Dym: Stationary measures for the flow of a linear differential equation driven by white noise, Trans. Amer. Math. Soc. 123 (1966), 130-164.

[ 3 ] T. Hida: Canonical representations of Gaussian processes and their applications, Mem. Coll. Sci. Univ. Kyoto, Ser. A, Math. 34 (1960), 109-155.

[ 4 ] L. Hörmander: Hypoelliptic second order differential equations, Acta Math. 119 (1967), 141-171.

[5] N. Levinson and H. P. McKean, Jr.: Weighted trigonometrical approximation on $\boldsymbol{R}^{1}$ with application to the germ field of a stationary Gaussian noise, Acta Math. 112 (1964), 99-143.

[6] Y. Okabe: Stationary Gaussian processes with Markovian property and M. Sato's hyperfunctions, to appear in Japanese J. of Math., 41 (1973), 69-122.

Nagoya University 